\title{
SQUAMOUS CELL CARCINOMA AS A LATE COMPLICATION OF CHRONIC OSTEOMYELITIS
}

\author{
Lakhey S*, Lakhey M*, Shrestha B P*
}

\section{ABSTRACT}

Squamous cell carcinoma is a rare but well documented complication of chronic osteomyelitis. With the advent of antibiotics, better health care delivery to the people and improving socioeconomic conditions, the incidence of squamous cell carcinoma arising as a late complication of acute haematogenous osteomyelitis has become increasingly less. Occasional difficulty in the differential diagnosis of a pseudoepitheliomatous hyperplasia and well differentiated squamous cell carcinoma can be troublesome. These two cases are presented to remind the clinician of this now increasingly rare complication of chronic osteomyelitis and the occasional diagnostic dilemma involved in its histopathological diagnosis.

\section{Key Words: Squamous Cell Carcinoma, Chronic Osteomyelitis.}

\section{INTRODUCTION}

Squamous cell carcinoma is a rare but well documented complication of osteomyelitis. ${ }^{1-9}$ Prompt and adequate treatment of acute haematogenous osteomyelitis can prevent this complication arising from the same. With the advent of antibiotics, better health care delivery to the people and improving socio-economic conditions, the incidence of squamous cell carcinoma arising as a late complication of acute haematogenous osteomyelitis will become increasingly less. However, it is a complication well worth remembering for an orthopaedic surgeon because if it is caught in the earlier stages, ablative surgery produces reliable cure.

\section{CASE \# 1}

Mr. SBR 54/M from the eastern hills of Nepal came to the Orthopaedic OPD of BPKIHS with complaints of a foul

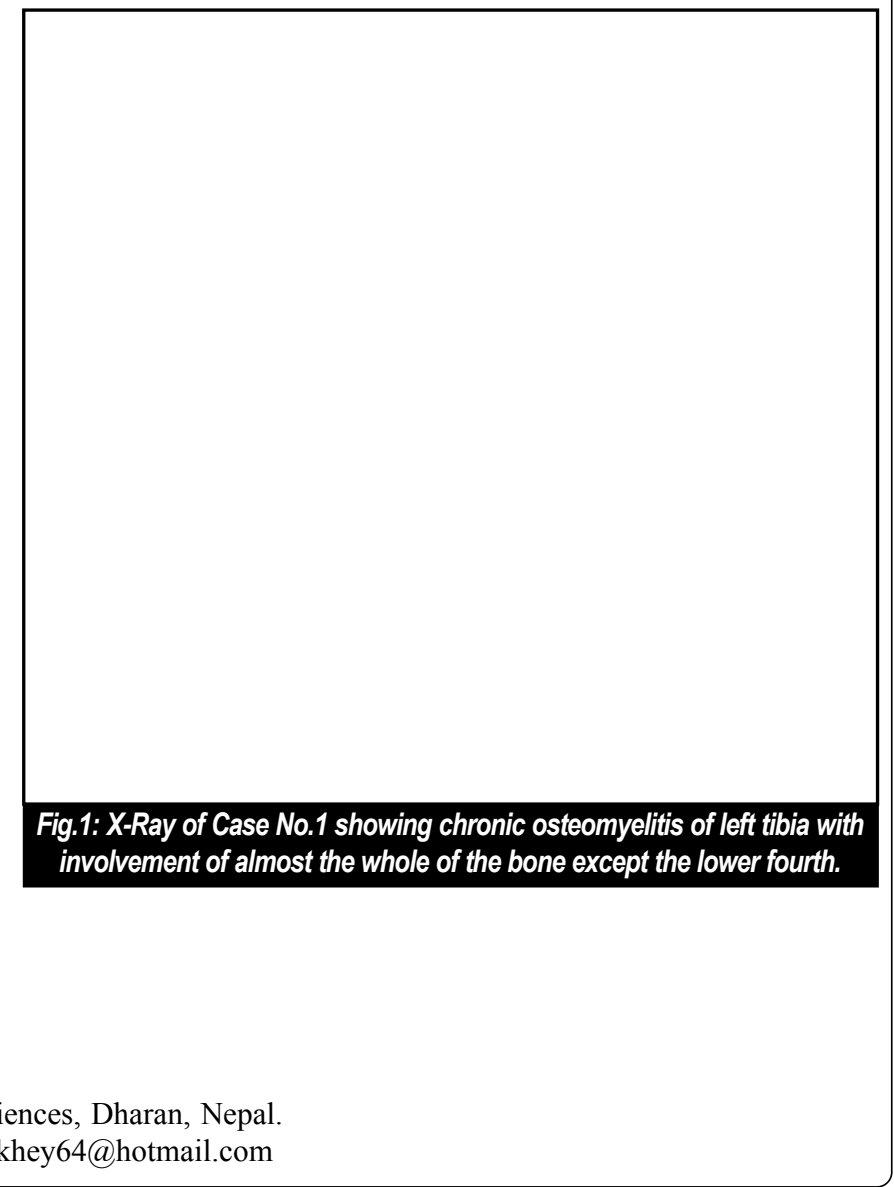




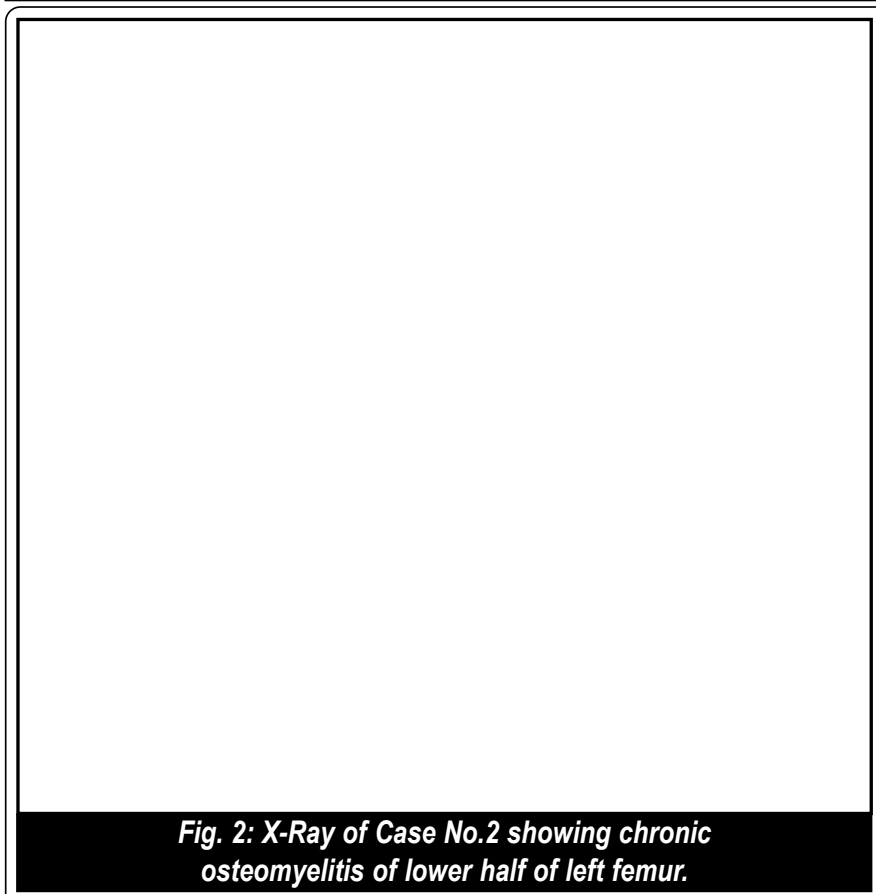

About 40 years ago, the patient developed features suggestive of acute haematogenous osteomyelitis of left tibia. Due to ignorance and lack of medical doctors nearby, he did not receive proper treatment, and the patient developed chronic osteomyelitis with discharging sinus from left tibia. The sinus healed to recur similarly again 5/6 times in the next 23 years. Around 17 years ago, the sinus started discharging pus and the tract did not close. The wound became so foul-swelling that his relatives and friends started staying away from him.

He came to British Military Hospital, Dharan where sequestrectomy curettage and sinus tract excision was done. He was relieved of his symptoms for some time but the foul smelling, discharging sinus and ulcer recurred. The ulcer did not heal with regular dressings. On examination, there was an ulcer about $6 \mathrm{~cm} \times 5 \mathrm{~cm} \times 3 \mathrm{~cm}$ over antero-medial aspect of upper third of left leg. The floor of the ulcer was dirty, the margins were everted and it was discharging foul-smelling, dirty colored fluid. X-ray showed evidence of chronic osteomyelitis of left tibia with involvement of almost the whole of the bone except the lower fourth (Fig.1). Biopsy from the ulcer margin including the adjacent tibia revealed features of pseudoepitheliomatous hyperplasia (PEH) of squamous epithelium. A repeat biopsy from deeper tissues showed well differentiated squamous cell carcinoma (Fig. $3 \& 4$ ). Chest xray showed no cannon-ball deposits and other haematological and biochemical parameters were within normal limits. An above knee amputation was done. A follow-up after $1 \frac{1}{2}$ years showed no metastasis.

\section{CASE \# 2}

Mr. KM 45/M from the eastern hills of Nepal presented with complaints of a non-healing ulcer in the lower left thigh for

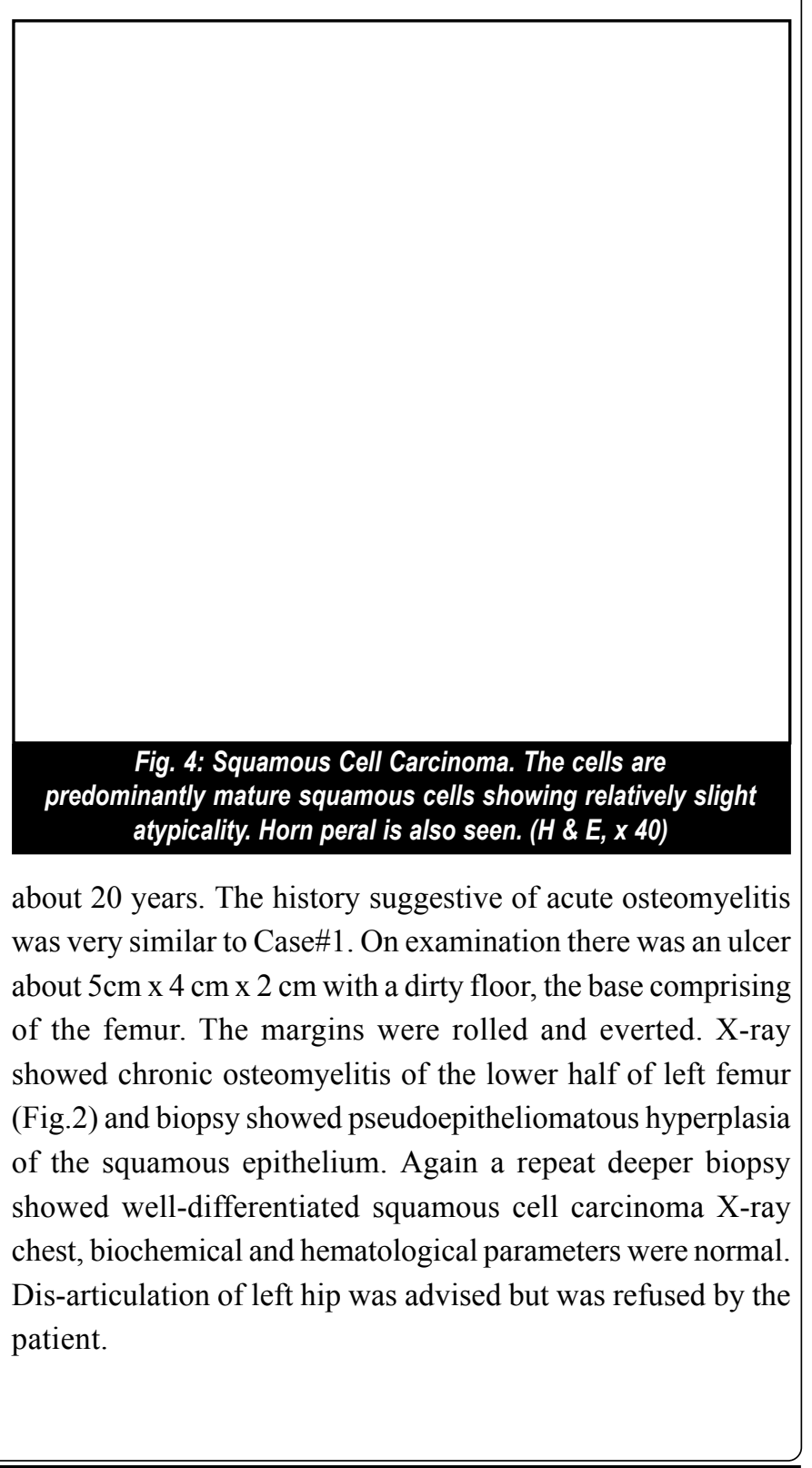




\section{DISCUSSION}

Squamous cell carcinoma (SCC) is a rare but well documented complication of osteomyelitis. ${ }^{1-9}$ The incidence of this rare complication has been estimated at $0.23-1.6 \%$ of all cases of chronic Osteomyelitis. ${ }^{5}$ The tumour develops after 4.5 to 50 years (average 30 years) of chronic infection with a discharging fistula. ${ }^{2,6}$ Epithelialisation of the fistulous tract has been demonstrated. The cancer arises in the dysplastic epithelium and can secondarily spread to the bone. Changes in the character of a previously innocuous osteomyelitis sinus tract should arouse suspicion of neoplastic transformation. ${ }^{7}$

Occasional difficulty in the differential diagnois of pseudoepitheliomatous hyperplasia (PEH) and welldifferentiated squamous cell carcinoma can be troublesome. Although the repeat biopsy too showed features similar to $\mathrm{PEH}$, on careful examination, features like individual cell keratinization, nuclear hyperplasia and hyperchromasia were evident. Invasion of the epithelial proliferations by leucocytes and disintegration of some of the epidermal cells, typically seen in PEH were not seen. Difficulties may be encountered in diagnosing malignant transformation especially whenever repeated deeper tissue layers biopsies may fail to reveal the true pathology. ${ }^{2}$ We faced this diagnostic difficulty in both the cases and repeat biopsies had to be done. Biopsy of various depths of the sinus including bone is essential for a correct diagnosis. Malignant transformation may be missed in a superficial biopsy. ${ }^{7}$

Inspite of all the above criteria taken into account it still may be very difficult to differentiate SCC from PEH from a single histologic section. Multiple biopsies and a detailed clinical history may be necessary for differentiation. Amputation meeting the criteria for oncological surgery has been the most reliable method of treatment of the tumour and chronic infection. However, current literature demonstrates that Mohs micrographic surgery ${ }^{8,9}$ offers a good therapeutic limb sparing option for control of locally invasive disease.

\section{REFERENCE} s. s.

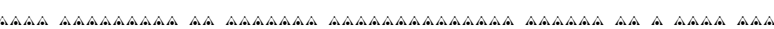

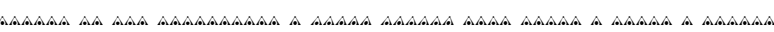

ش.

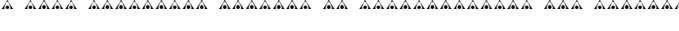

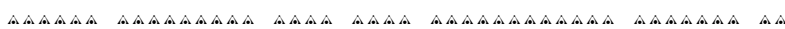

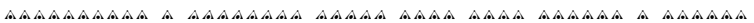
$\therefore$

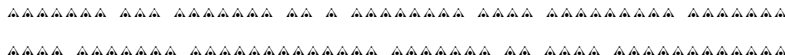

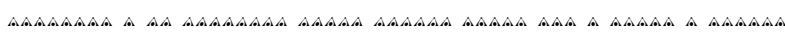
O $\mathrm{O} O$ 